\title{
Response prediction of hepatocellular carcinoma undergoing transcatheter arterial chemoembolization: unlocking the potential of CT texture analysis through nested decision tree models
}

\author{
Jan Vosshenrich ${ }^{1}$ (D) Christoph J. Zech ${ }^{1} \cdot$ Tobias Heye $^{1} \cdot$ Tuyana Boldanova $^{2,3} \cdot$ Geoffrey Fucile $^{4} \cdot$ Stefan Wieland $^{3}$. \\ Markus H. Heim ${ }^{2,3}$ • Daniel T. Boll ${ }^{1}$
}

Received: 2 September 2020 / Accepted: 11 November 2020 / Published online: 3 December 2020

(C) The Author(s) 2020

\begin{abstract}
Objectives To investigate if nested multiparametric decision tree models based on tumor size and CT texture parameters from pre-therapeutic imaging can accurately predict hepatocellular carcinoma (HCC) lesion response to transcatheter arterial chemoembolization (TACE).

Materials and methods This retrospective study (January 2011-September 2017) included consecutive pre- and post-therapeutic dynamic CT scans of 37 patients with 92 biopsy-proven HCC lesions treated with drug-eluting bead TACE. Following manual segmentation of lesions according to modified Response Evaluation Criteria in Solid Tumors criteria on baseline arterial phase CT images, tumor size and quantitative texture parameters were extracted. HCCs were grouped into lesions undergoing primary TACE (VT-lesions) or repeated TACE (RT-lesions). Distinct multiparametric decision tree models to predict complete response (CR) and progressive disease (PD) for the two groups were generated. AUC and model accuracy were assessed.

Results Thirty-eight of 72 VT-lesions (52.8\%) and 8 of 20 RT-lesions (40\%) achieved CR. Sixteen VT-lesions (22.2\%) and 8 RT-lesions (40\%) showed PD on follow-up imaging despite TACE treatment. Mean of positive pixels (MPP) was significantly higher in VT-lesions compared to RT-lesions (180.5 vs 92.8, $p=0.001)$. The highest AUC in ROC curve analysis and accuracy was observed for the prediction of CR in VT-lesions (AUC 0.96, positive predictive value 96.9\%, accuracy 88.9\%). Prediction of PD in VT-lesions (AUC 0.88, accuracy 80.6\%), CR in RT-lesions (AUC 0.83, accuracy 75.0\%), and PD in RT-lesions (AUC 0.86, accuracy 80.0\%) was slightly inferior.

Conclusions Nested multiparametric decision tree models based on tumor heterogeneity and size can predict HCC lesion response to TACE treatment with high accuracy. They may be used as an additional criterion in the multidisciplinary treatment decision-making process.

Key Points

- HCC lesion response to TACE treatment can be predicted with high accuracy based on baseline tumor heterogeneity and size.

- Complete response of HCC lesions undergoing primary TACE was correctly predicted with $88.9 \%$ accuracy and a positive predictive value of $96.9 \%$.

- Progressive disease was correctly predicted with $80.6 \%$ accuracy for lesions undergoing primary TACE and $80.0 \%$ accuracy for lesions undergoing repeated TACE.
\end{abstract}

Jan Vosshenrich

jan.vosshenrich@usb.ch

1 Department of Radiology, University Hospital Basel, Petersgraben 4, 4031 Basel, Switzerland

2 Clarunis - University Center for Gastrointestinal and Liver Diseases, Petersgraben 4, 4031 Basel, Switzerland

3 Department of Biomedicine, University Hospital Basel, University of Basel, Hebelstrasse 20, 4031 Basel, Switzerland

4 sciCORE - Center for Scientific Computing, University of Basel, Klingelbergstrasse 50/70, 4031 Basel, Switzerland 
Keywords Hepatocellular carcinoma $\cdot$ Treatment outcome $\cdot$ X-ray computed tomography $\cdot$ Therapeutic chemoembolization · Decision trees

$\begin{array}{ll}\text { Abbreviations } \\ \text { BCLC } & \text { Barcelona Clinic Liver Cancer } \\ \text { CR } & \text { Complete response } \\ \text { HCC } & \text { Hepatocellular carcinoma } \\ \text { MPP } & \text { Mean of positive pixels } \\ \text { PD } & \text { Progressive disease } \\ \text { RT-lesions } & \text { Previously treated lesions undergoing repeated } \\ & \text { TACE } \\ \text { TACE } & \text { Transcatheter arterial chemoembolization } \\ \text { VT-lesions } & \text { Virgin lesions undergoing primary TACE }\end{array}$

\section{Introduction}

Hepatocellular carcinoma (HCC) which accounts for more than $90 \%$ of primary liver cancers is the sixth most common cancer regarding incidence and the fourth most common cause of cancer-related mortality worldwide [1-3]. In accordance with clinical practice guidelines, patients diagnosed with intermediate or advanced stage of neoplastic disease are not amenable to curative surgical resection but are allocated to loco-regional interventional treatment or, alternatively, protein kinase inhibitor therapy such as sorafenib [3, 4]. Particularly in Barcelona Clinic Liver Cancer (BCLC) stage $\mathrm{B}$ patients, transcatheter arterial chemoembolization (TACE) represents the standard of care in many institutions $[3,5,6]$. Furthermore, TACE is also the most widely used bridging therapy in BCLC stage A patients awaiting liver transplantation. The efficacy of TACE has been demonstrated in randomized control trials $[7,8]$. Noticeable differences in overall survival however suggest that not all treated lesions will effectively respond to TACE, as HCC patients show a wide spectrum of potential short- and long-term outcomes following treatment [9].

In recent years, efforts have been made identifying biomarkers of HCCs potentially predicting lesion response to TACE treatment, aiming to facilitate a decision process whether a patient should undergo primary or repeated TACE or be treated by different means. Utilized predictive algorithms were based on either laboratory results and clinical scores [10] or imaging parameters derived from CT or MRI texture analysis $[11,12]$. The advent of such concepts demonstrated the emerging trend towards precision medicine in patients with focal liver disease and depicted the general ability to extend the assessment of HCC lesions beyond current classification systems.

While already suggested prediction approaches take several parameters into account, they are generally only used individually and dichotomously. By nesting multiple factors into a decision tree model, potentially using varying thresholds of redundant factors at different locations within the decision tree, a further increase in accuracy of treatment response prediction may result.

The aim of our study was to investigate the value of histogram-based CT texture analysis-derived nested decision tree models for the prediction of HCC lesion response to TACE treatment according to modified Response Evaluation Criteria in Solid Tumors (mRECIST) criteria in order to demonstrate that accurate prediction of complete response and progressive disease prior to both primary and repeated TACE is feasible.

\section{Materials and methods}

\section{Study sample}

This retrospective study was approved by the institutional review board; patients gave written informed consent. All patients treated with TACE and histopathologically proven HCC during the observation window between January 2011 and September 2017 were included. Exclusion criteria were (1) patients without baseline dynamic contrast-enhanced CT of the liver, (2) lack of follow-up CT imaging at the earliest 4 weeks after treatment, and (3) patients with non-diagnostic CT images. The final study sample consisted of 37 patients with a total of 92 individually treated HCC lesions (Fig. 1).

Both BCLC stage B patients undergoing palliative TACE and BCLC stage A patients being poor candidates for surgery or receiving TACE as a bridging treatment while awaiting liver transplantation were included. Due to the initial heterogeneity of disease stages, this study solely focused on target response prediction according to $\mathrm{mRECIST}$ and did not assess long-term outcome parameters, e.g., overall survival.

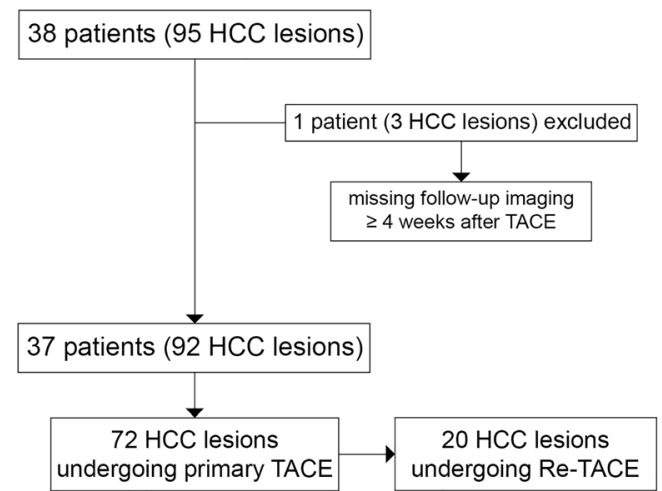

Fig. 1 Flowchart of the study sample including exclusion criteria and grouping of lesions 


\section{CT imaging}

All patients underwent four-phase CT according to the institutional standard liver imaging protocol. Imaging acquisitions were as follows: unenhanced, late arterial phase (AP), portal venous phase (PVP) and 3-min delayed phase (DP). CT examinations were acquired on a 128 -slice (Somatom Definition Edge, Siemens Healthineers; tube settings $100 \mathrm{kV}, 110$ eff. mAs; collimation $128 \mathrm{~mm} \times 0.6$ $\mathrm{mm}$, pitch 0.6 , rotation time $0.5 \mathrm{~s}$, slice thickness $1.5 \mathrm{~mm}$ ) or 256-slice (Somatom Definition Flash, Siemens Healthineers; tube settings $100 \mathrm{kV}, 65$ eff. mAs; collimation $128 \mathrm{~mm} \times 0.6 \mathrm{~mm}$, pitch 0.6 , rotation time $0.5 \mathrm{~s}$, slice thickness $1.5 \mathrm{~mm}$ ) scanner system. Following the unenhanced scan, $1.2 \mathrm{ml} / \mathrm{kg}$ of $370 \mathrm{mg} \mathrm{I} / \mathrm{ml}$ iopromide (Ultravist ${ }^{\circledR}$ 370, Bayer Pharma) was injected intravenously at a flow rate of $4 \mathrm{ml} / \mathrm{s}$ by a power injector (Ulrich Medical). Using bolus tracking technique, AP images were acquired $18 \mathrm{~s}$ after reaching $100 \mathrm{HU}$ in the descending aorta at the level of the celiac trunk. PVP and DP images were obtained $70 \mathrm{~s}$ and $180 \mathrm{~s}$ after reaching the scan initiation threshold. Timepoints of CT examinations were as follows: baseline imaging within 1 week prior to TACE, first follow-up imaging at 4 weeks after TACE and subsequent follow-ups at 3-month intervals.

\section{Transcatheter arterial chemoembolization procedure}

TACE procedures were performed by one of two interventional radiologists with $>10$ years of interventional experience (C.J.Z.). The right femoral artery was punctured using Seldinger technique, and a 4-French cobra or sidewinder catheter was inserted into the celiac trunk and common hepatic artery, respectively. In case of variant vascular anatomy, the superior mesenteric artery was catheterized additionally. To visualize feeding arteries of the tumor, digital subtraction angiography was performed and feeding vessels were superselectively intubated with a highly flexible 2.7-French microcatheter (ProGreat, Terumo). For embolization, doxorubicin-coated $100-\mu \mathrm{m}$ beads (Tandem Beads, Embozene, now Boston Scientific) were slowly injected under fluoroscopic guidance up to a maximum dose of $150 \mathrm{mg}$ doxorubicin. If stasis in the feeding vessel and disappearance of tumor staining was observed earlier, injection was terminated at a lower total doxorubicin dose. A closing DynaCT was obtained to assess treatment success.

\section{Image and texture analysis}

Image datasets of all patients and timepoints were imported into mint Lesion ${ }^{\text {TM }} 3.0$ software (Mint Medical GmbH; commercially available) for post-processing. Every lesion was manually segmented on arterial phase images (axial plane, slice thickness $1.5 \mathrm{~mm}$ ) according to mRECIST criteria by one radiologist with 2 years of experience in abdominal imaging (J.V.). All segmentations were reviewed by one radiologist specialized in abdominal imaging with $>15$ years of experience (D.T.B.) and one radiologist specialized in abdominal imaging and interventional radiology with $>15$ years of experience (C.J.Z.). Measurement discrepancies were resolved by consensus. CT texture analysis of segmented regions of interest (ROIs) was performed automatically by mint Lesion $^{\mathrm{TM}}$ software based on gray-level histograms and included the following parameters: entropy, kurtosis, skewness, mean of positive pixels (MPP), and uniformity of positive pixel (UPP) distribution.

Response to TACE treatment as per mRECIST criteria was calculated after manual segmentation of lesions' enhancing portions on baseline and follow-up CT examinations; mRECIST timepoint response evaluation criteria were the following: complete response (CR), disappearance of any intratumoral arterial enhancement; partial response (PR), at least $30 \%$ decrease in the sum of diameters of viable tumor; stable disease (SD), any cases not qualifying for either partial response or progressive disease; and progressive disease (PD), an increase of at least $20 \%$ in the sum of the diameters of viable tumor. If a lesion did not achieve CR after TACE and the institutional multidisciplinary gastrointestinal tumor board decided for repeated TACE treatment, a new baseline was set for the remaining enhancing portions of the lesion. This new baseline was used for subsequent response assessment after repeated TACE.

All CT texture analysis parameters, mRECIST timepoint responses, and lesion measurements specifically short- and long-axis diameters (in $\mathrm{mm}$ ) and area (in $\mathrm{mm}^{2}$ ) were extracted.

\section{Statistical analysis and graphical visualization}

Data was analyzed using SPSS 14 (IBM Corporation) for descriptive statistics and JMP® 14.0 (SAS Institute, Inc.) for calculation of prediction models, both commercially available.

HCCs were divided into two groups: (1) previously untreated lesions undergoing primary TACE (VT-lesions) and (2) lesions receiving repeated TACE (RT-lesions) due to incomplete response or progressive disease after the first TACE. This step was performed in order to differentiate if the first treatment sequence has any effect on the texture of the remaining viable tumor portions, and thus, a change in parameter thresholds needed for accurate response prediction. Baseline characteristics were compared using an independent Student's $t$ test, chi-square test, and Fisher's exact test (significance level $p<0.05)$. 
The two datasets were imported in the Prediction Profiler module in JMP®. CR or PD was defined as outcome parameters. The module created varying testing and confirmation datasets during the modeling process and calculated prediction models based on tumor size (area), surrounding hepatic parenchyma (cirrhotic vs non-cirrhotic liver), and parameters from CT texture analysis, resulting in four different decision tree models with automatically generated optimal discrimination thresholds for the respective parameters in each model: (1) VT-lesions with CR as goal of prediction, (2) VT-lesions with PD as goal of prediction, (3) RT-lesions with CR as goal of prediction, and (4) RT-lesions with PD as goal of prediction. Categorization of lesions was based on binary splitting. The minimum split size at each node was set at ten lesions to avoid overfitting.

Parameters' contribution to the model, receiver operating characteristic (ROC) curve, and confusion matrices to depict model performance were generated. Based on confusion tables, positive prediction value (PPV), negative prediction value (NPV), and accuracies were calculated.

\section{Results}

\section{Baseline characteristics}

Of the 92 included lesions, 17 were found in women and 75 in men. Mean age at baseline imaging was 70.3 years ( \pm 9.3 , range 49-88). Eighty-seven percent (80/92) of lesions arose from cirrhotic livers, while $13 \%$ (12/92) were located in non-cirrhotic liver parenchyma. The mean number of HCC lesions per patient was 3 (range 1-7). Distribution of lesions between the different anatomical liver segments was heterogenous, and the majority was found in the right liver lobe $(67 / 92,67.8 \%)$, especially in segment VIII $(31 / 92,33.7 \%)$. All baseline

Table 1 Baseline characteristics of VT-lesions and RT-lesions showing complete or non-complete response to TACE

\begin{tabular}{|c|c|c|c|c|c|c|c|}
\hline & \multicolumn{3}{|l|}{ VT-lesions } & \multicolumn{3}{|l|}{ RT-lesions } & \multirow[t]{2}{*}{ All lesions (total) } \\
\hline & $\mathrm{CR}$ & Non-CR & Total & $\mathrm{CR}$ & Non-CR & Total & \\
\hline No. of HCC lesions & 38 & 34 & 72 & 8 & 12 & 20 & 92 \\
\hline Age (range) & $69.7(61-84)$ & 73.9 (49-87) & 71.7 (49-87) & $64.0(51-80)$ & $66.1(49-88)$ & $65.3(49-88)$ & $70.3(49-88)$ \\
\hline \multicolumn{8}{|l|}{ Gender (\%) } \\
\hline Women & $10(26.3)$ & $5(14.7)$ & $15(20.8)$ & $1(12.5)$ & $1(8.3)$ & $2(10)$ & $17(18.5)$ \\
\hline Men & $28(73.7)$ & $29(85.3)$ & $57(79.2)$ & $7(87.5)$ & $11(91.7)$ & $18(90)$ & $75(81.5)$ \\
\hline \multicolumn{8}{|l|}{ Cirrhosis (\%) } \\
\hline Yes & $37(97.4)$ & $23(67.6)$ & $60(83.3)$ & $8(100)$ & $12(100)$ & $20(100)$ & $80(87)$ \\
\hline No & $1(2.6)$ & $11(32.4)$ & $12(16.7)$ & $0(0)$ & $0(0)$ & $0(0)$ & $12(13)$ \\
\hline \multicolumn{8}{|l|}{ BCLC stage (\%) } \\
\hline $\mathrm{A}$ & $22(57.9)$ & $16(47.1)$ & $38(52.8)$ & $2(25)$ & $8(66.7)$ & $10(50)$ & $48(52.2)$ \\
\hline B & $16(42.1)$ & $18(52.9)$ & $34(47.2)$ & $6(75)$ & $4(33.3)$ & $10(50)$ & $44(47.8)$ \\
\hline \multicolumn{8}{|c|}{ No. of HCC lesions per patient } \\
\hline $\mathrm{I}$ & $3.1(1-7)$ & $2.3(1-7)$ & $2.7(1-7)$ & $2(1-4)$ & $1.5(1-2)$ & $1.7(1-4)$ & $2.5(1-7)$ \\
\hline II & $0(0)$ & $1(2.9)$ & $1(1.4)$ & $0(0)$ & $0(0)$ & $0(0)$ & $1(1.1)$ \\
\hline III & $5(13.2)$ & $5(14.7)$ & $10(13.9)$ & $0(0)$ & $0(0)$ & $0(0)$ & $10(10.9)$ \\
\hline IVa & $1(2.6)$ & $1(2.9)$ & $2(2.8)$ & $0(0)$ & $0(0)$ & $0(0)$ & $2(2.2)$ \\
\hline $\mathrm{IVb}$ & $2(5.3)$ & $3(8.8)$ & $5(6.9)$ & $1(12.5)$ & $2(16.7)$ & $3(15)$ & $8(8.7)$ \\
\hline \multicolumn{8}{|l|}{ Liver segment $(\%)$} \\
\hline Left lobe & $1(2.6)$ & $3(8.8)$ & $4(5.6)$ & $0(0)$ & $0(0)$ & $0(0)$ & $4(4.3)$ \\
\hline V & $9(23.7)$ & $13(38.2)$ & $22(30.6)$ & $1(12.5)$ & $2(16.7)$ & $3(15)$ & $25(27.2)$ \\
\hline VI & $9(23.7)$ & $2(5.9)$ & $11(15.3)$ & $1(12.5)$ & $2(16.7)$ & $3(15)$ & $14(15.2)$ \\
\hline VII & $3(7.9)$ & $5(14.7)$ & $8(11.1)$ & $0(0)$ & $0(0)$ & $0(0)$ & $8(8.7)$ \\
\hline VIII & $9(23.7)$ & $4(11.8)$ & $13(18.1)$ & $0(0)$ & $1(8.3)$ & $1(5)$ & $14(15.2)$ \\
\hline Right lobe & $8(21.1)$ & $10(29.4)$ & $18(25.0)$ & $6(75)$ & $7(58.3)$ & $13(65)$ & $31(33.7)$ \\
\hline Area in $\mathrm{mm}^{2}(\mathrm{SD})$ & $29(76.3)$ & $21(61.8)$ & $50(69.4)$ & $7(87.5)$ & $10(83.3)$ & $17(85)$ & $67(67.8)$ \\
\hline $\begin{array}{l}\text { Long axis in } \mathrm{mm} \\
\text { (SD) }\end{array}$ & $279.4( \pm 385.2)$ & $1938.3( \pm 3131.2)$ & $1062.8( \pm 2308.6)$ & $659.4( \pm 864.3)$ & $876.9( \pm 1362.8)$ & $789.9( \pm 1167.2)$ & $1003.4( \pm 2110.8)$ \\
\hline MPP (SD) & $20.3( \pm 11.2)$ & $49.2( \pm 34.1)$ & $33.9( \pm 28.6)$ & $30.4( \pm 19.5)$ & $41.2( \pm 31.0)$ & $36.9( \pm 26.9)$ & $34.6( \pm 28.1)$ \\
\hline Entropy (SD) & $248.2( \pm 277.8)$ & $104.8( \pm 21.9)$ & $180.5( \pm 213.6)$ & $95.3( \pm 26.5)$ & $91.1( \pm 18.8)$ & $92.8( \pm 21.6)$ & $161.4( \pm 192.4)$ \\
\hline Kurtosis (SD) & $5.921( \pm 0.61)$ & $6.265( \pm 0.41)$ & $6.084( \pm 0.55)$ & $5.724( \pm 0.94)$ & $6.076( \pm 0.68)$ & $5.934( \pm 0.79)$ & $6.051( \pm 0.61)$ \\
\hline Skewness (SD) & $3.0( \pm 1.2)$ & $3.6( \pm 0.9)$ & $3.3( \pm 1.1)$ & $3.2( \pm 0.7)$ & $3.5( \pm 1.1)$ & $3.4( \pm 1.0)$ & $3.3( \pm 1.1)$ \\
\hline Uniformity (SD) & $0.0( \pm 0.36)$ & $-0.1( \pm 0.35)$ & $-0.1( \pm 0.35)$ & $0.1( \pm 0.34)$ & $-0.2( \pm 0.40)$ & $-0.1( \pm 0.4)$ & $0.1( \pm 0.36)$ \\
\hline Area in $\mathrm{mm}^{2}(\mathrm{SD})$ & $0.0206( \pm 0.009)$ & $0.0160( \pm 0.005)$ & $0.0184( \pm 0.008)$ & $0.0268( \pm 0.022)$ & $0.0191( \pm 0.009)$ & $0.0222( \pm 0.015)$ & $0.0192( \pm 0.010)$ \\
\hline
\end{tabular}

$V T$-lesions lesions undergoing primary TACE, RT-lesions lesions undergoing repeated TACE, CR complete response, Non-CR non-complete responders, $S D$ standard deviation, TACE transcatheter arterial chemoembolization 
characteristics are summarized in Table 1. Seventy-two HCC lesions in our study were treated with a single TACE, while 20 tumors required repeated treatment sessions (range 2-4 TACE treatments).

MPP was significantly higher in VT-lesions compared to RT-lesions $(180.5 \pm 213.6$ vs $92.8 \pm 21.6, p=0.001)$. No other significant differences in baseline $\mathrm{CT}$ texture parameters were observed. Data is summarized in Table 2.

\section{Response to TACE Treatment}

In the primary TACE group, 38 of the 72 lesions (52.8\%) showed CR on post-therapeutic imaging (example in Fig. 2); 16 lesions (22.2\%) showed PD on follow-up CT. In the repeated TACE group, 8 of 20 lesions $(40 \%)$ were rated as $\mathrm{CR}$, while 8 lesions (40\%) showed PD on follow-up imaging.

The course of each lesion over time and target response at follow-up imaging timepoints is illustrated as a swimmer plot (supplemental material). Mean time frame for overall disease follow-up was significantly longer for VT-lesions, compared to RT-lesions ( $268 \pm 235$ days vs $171 \pm 110$ days, $p=0.01$ ). Timepoints of disease-related death or liver transplantation are visualized in the swimmer plot.

\section{Prediction of lesion response to primary TACE}

The calculated decision tree model to predict CR in VTlesions had eight binary splits and used six parameters to
Table 2 Comparison of baseline characteristics between RTlesions and VT-lesions

\begin{tabular}{|c|c|c|c|}
\hline & VT-lesions & RT-lesions & $p$ value \\
\hline No. of HCC lesions & 72 & 20 & - \\
\hline Age (range) & $71.7(49-87)$ & $65.3(49-88)$ & $0.03 *$ \\
\hline \multicolumn{4}{|l|}{ Gender $(\%)$} \\
\hline Women & $15(20.8)$ & $2(10)$ & \multirow[t]{2}{*}{0.27} \\
\hline Men & $57(79.2)$ & $18(90)$ & \\
\hline \multicolumn{4}{|l|}{ Cirrhosis $(\%)$} \\
\hline Yes & $60(83.3)$ & $20(100)$ & \multirow[t]{2}{*}{$<0.001^{*}$} \\
\hline No & $12(16.7)$ & $0(0)$ & \\
\hline \multicolumn{4}{|l|}{ BCLC stage (\%) } \\
\hline A & $38(52.8)$ & $10(50)$ & \multirow[t]{2}{*}{0.83} \\
\hline $\mathrm{B}$ & $34(47.2)$ & $10(50)$ & \\
\hline No. of HCC lesions per patient & $2.7(1-7)$ & $1.7(1-4)$ & $<0.001 *$ \\
\hline Follow-up after TACE in days (SD) & $268( \pm 235)$ & $171( \pm 110)$ & \multirow[t]{6}{*}{$<0.001 *$} \\
\hline I & $1(1.4)$ & $0(0)$ & \\
\hline II & $10(13.9)$ & $0(0)$ & \\
\hline III & $2(2.8)$ & $0(0)$ & \\
\hline IVa & $5(6.9)$ & $3(15)$ & \\
\hline $\mathrm{IVb}$ & $4(5.6)$ & $0(0)$ & \\
\hline \multicolumn{4}{|l|}{ Liver segment (\%) } \\
\hline Left lobe & $22(30.6)$ & $3(15)$ & \multirow[t]{6}{*}{$0.03 *$} \\
\hline $\mathrm{V}$ & $11(15.3)$ & $3(15)$ & \\
\hline VI & $8(11.1)$ & $0(0)$ & \\
\hline VII & $13(18.1)$ & $1(5)$ & \\
\hline VIII & $18(25.0)$ & $13(65)$ & \\
\hline Right lobe & $50(69.4)$ & $17(85)$ & \\
\hline Area in $\mathrm{mm}^{2}(\mathrm{SD})$ & $1062.8( \pm 2308.6)$ & $789.9( \pm 1167.2)$ & 0.61 \\
\hline Long axis in mm (SD) & $33.9( \pm 28.6)$ & $36.9( \pm 26.9)$ & 0.68 \\
\hline MPP (SD) & $180.5( \pm 213.6)$ & $92.8( \pm 21.6)$ & $0.001 *$ \\
\hline Entropy (SD) & $6.084( \pm 0.55)$ & $5.934( \pm 0.79)$ & 0.33 \\
\hline Kurtosis (SD) & $3.3( \pm 1.1)$ & $3.4( \pm 1.0)$ & 0.72 \\
\hline Skewness (SD) & $-0.1( \pm 0.35)$ & $-0.1( \pm 0.4)$ & 0.42 \\
\hline Uniformity (SD) & $0.0184( \pm 0.008)$ & $0.0222( \pm 0.015)$ & 0.31 \\
\hline
\end{tabular}

Statistically significant differences are marked by an asterisk

$V T$-lesions lesions undergoing primary TACE, RT-lesions lesions undergoing repeated TACE, SD standard deviation, TACE transcatheter arterial chemoembolization 

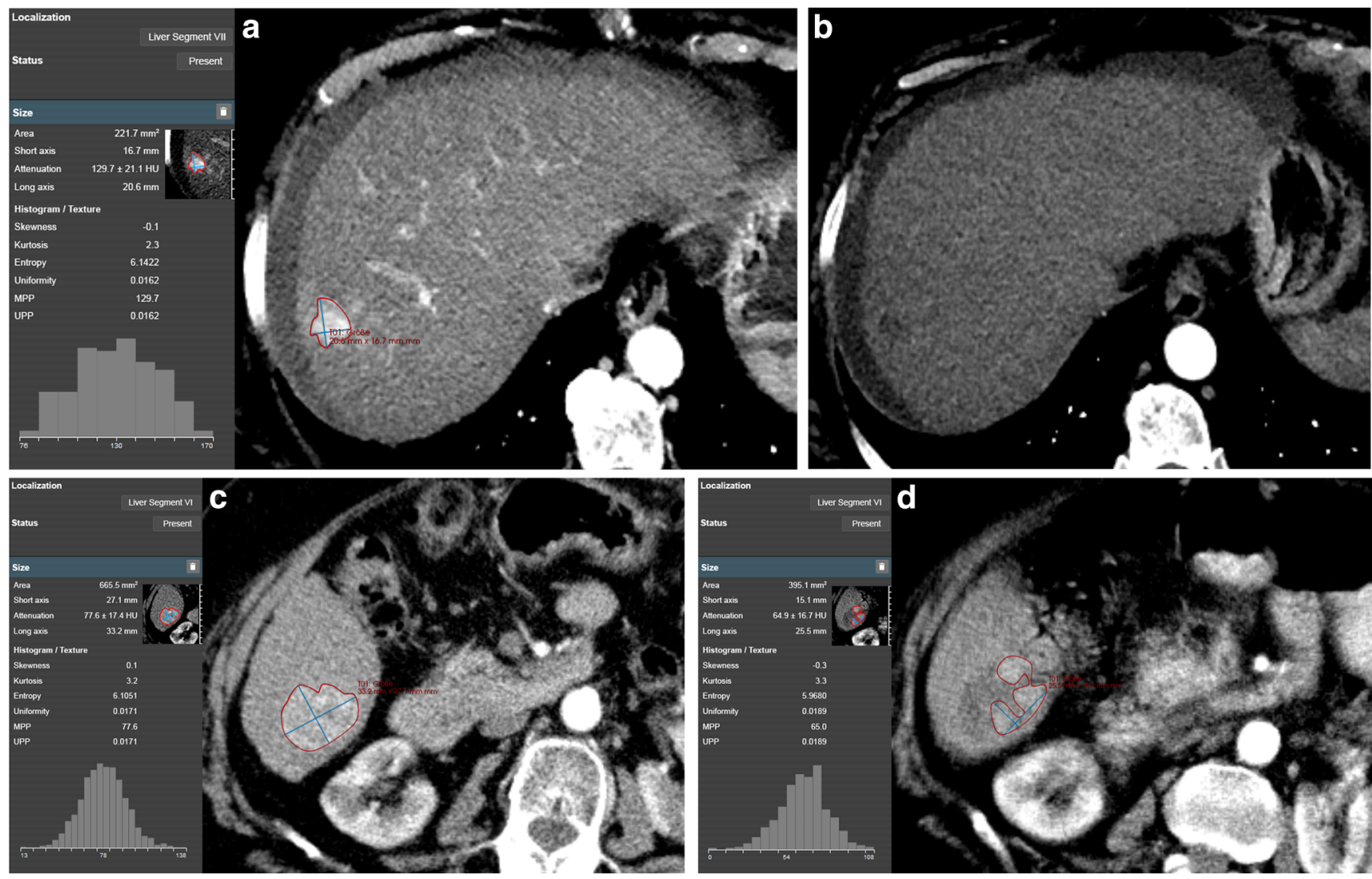

d
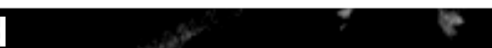

Fig. 2 a Screenshot of a manually segmented HCC lesion according to mRECIST criteria on baseline arterial phase imaging in mint Lesion ${ }^{\mathrm{TM}}$ 3.0 software with automatic generation of gray-level histograms and extraction of CT texture parameters. Favorable lesion size and parameters from CT texture analysis (especially mean of positive pixels (MPP) and uniformity) render this HCC lesion likely to respond completely to transcatheter arterial chemoembolization (TACE) treatment. b Four weeks after TACE treatment, the HCC shows no enhancement in arterial phase (thus, no viability) resulting in complete response as per mRECIST

criteria. c Another manually segmented HCC lesion at baseline imaging. Unfavorable lesion size, surrounding liver parenchyma (cirrhotic), and parameters from CT texture analysis (especially MPP and uniformity) at baseline imaging result in a low likelihood of complete response to TACE treatment. d Follow-up imaging 4 weeks after TACE treatment shows substantial residual arterial enhancement (= viable portions of the tumor), resulting in partial response as per mRECIST criteria. The patient subsequently underwent repeated TACE, and the post-treatment CT images were used as new baseline

a

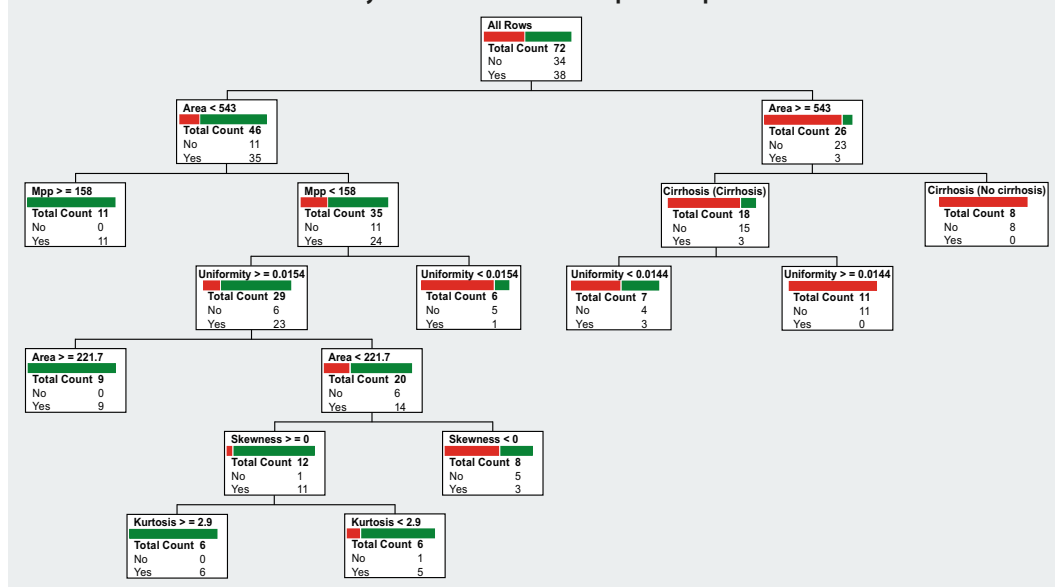

b

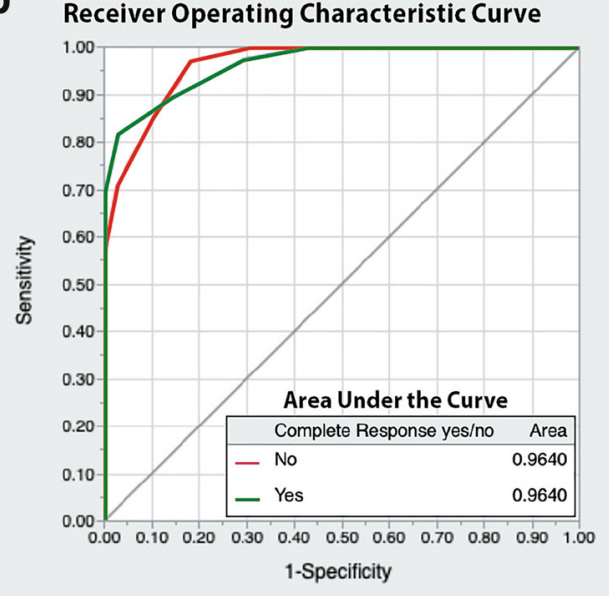

Fig. 3 a Decision tree model based on texture parameters, size, and surrounding liver parenchyma (cirrhotic vs non-cirrhotic) to predict complete response prior to primary transcatheter arterial chemoembolization (TACE) treatment. b ROC curve with AUC values for the model 

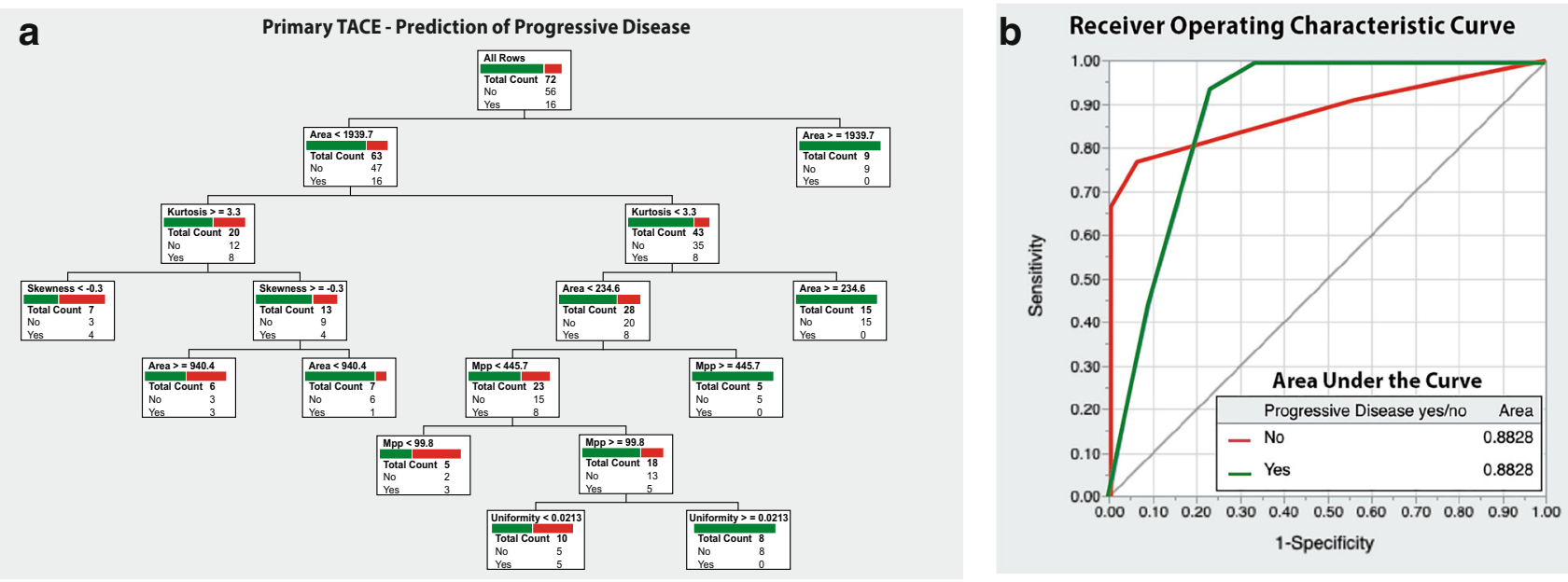

Fig. 4 a Decision tree model based on texture parameters, size, and surrounding liver parenchyma (cirrhotic vs non-cirrhotic) to predict progressive disease prior to primary transcatheter arterial chemoembolization (TACE) treatment. b ROC curve with AUC values for the model

accurately categorize lesions. These were as follows: area (total effect 0.744$)$, uniformity (0.267), cirrhosis $(0.145)$, MPP (0.019), skewness (0.003), and kurtosis (0.001). The model's ROC curve analysis reached an area under the curve (AUC) of 0.96. Correct prediction was achieved in 64 of 72 cases, resulting in a PPV of 96.9\%, an NPV of 82.5, and an accuracy of $88.9 \%$. The model is visualized in Fig. 3.

The decision tree model to predict PD in VT-lesions had seven binary splits and used five contributing parameters: area (0.974), kurtosis (0.201), skewness (0.078), MPP (0.004), and uniformity (0.003). ROC analysis reached an AUC of 0.88 . Correct target response was predicted for 58 of 72 lesions (PPV 58.3\%, NPV 85.0\%, resultant accuracy $80.6 \%$ ). The model is visualized in Fig. 4.

\section{Prediction of lesion response to Re-TACE}

The decision tree model to predict CR in RT-lesions had three binary splits with only one contributing parameter (area; total effect 0.986). The model's AUC was 0.83 . Correct target response was predicted in 15 of 20 lesions (PPV 80.0, NPV 83.3\%, resultant accuracy $75.0 \%$ ). The model is visualized in Fig. 5.

The decision tree model to predict PD in RT-lesions had three binary splits and three contributing parameters: area (0.97), MPP (0.125), and kurtosis (0.068). The AUC of the model was 0.86 , and correct response was predicted for 16 of 20 lesions (PPV 83.3\%, NPV 78.6\%, resultant accuracy $80.0 \%$ ). The model is visualized in Fig. 6. Parameter effects for each model are listed in Table 3.

\section{Discussion}

The aim of our study was to assess the feasibility of generating decision tree models based on pre-therapeutic CT texture parameters to predict complete response or progressive disease of HCC lesions to TACE treatment according to mRECIST criteria. Our results demonstrate that both target responses can be predicted with high accuracy for HCCs undergoing both primary TACE and repeated a

Repeated TACE - Prediction of Complete Response

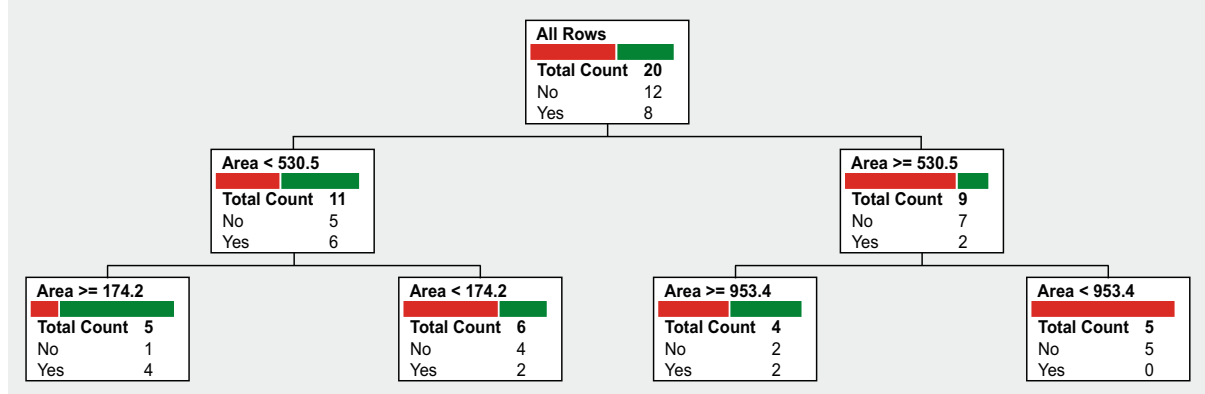

b

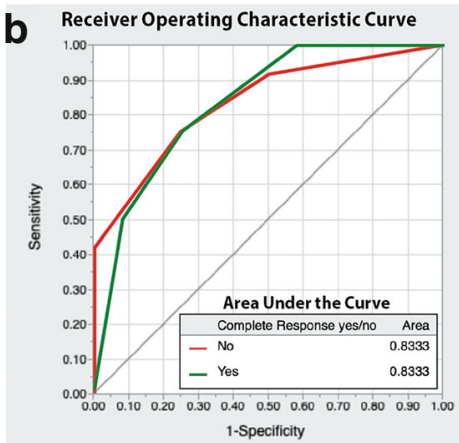

Fig. 5 a Decision-tree model based on texture parameters, size, and surrounding liver parenchyma (cirrhotic vs non-cirrhotic) to predict complete response prior to repeated transcatheter arterial chemoembolization (TACE) treatment. b ROC curve with AUC values for the model 

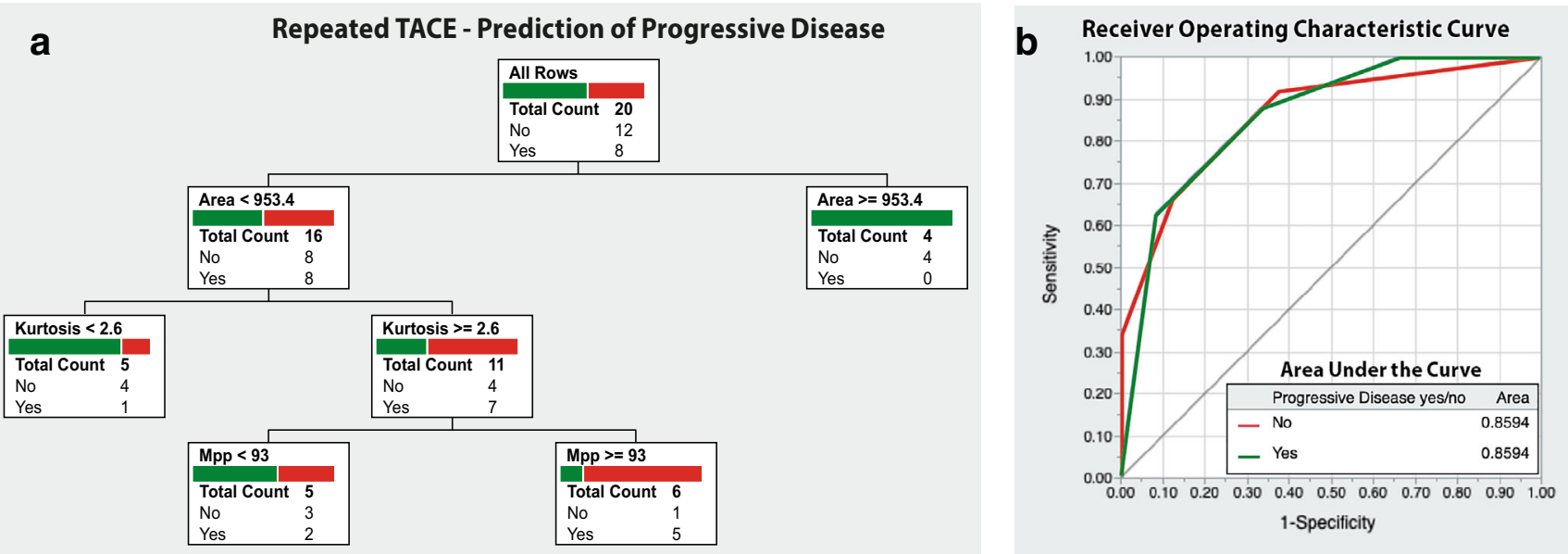

Fig. 6 a Decision-tree model based on texture parameters, size, and surrounding liver parenchyma (cirrhotic vs non-cirrhotic) to predict progressive disease prior to repeated transcatheter arterial chemoembolization (TACE) treatment. b ROC curve with AUC values for the model

TACE. To our knowledge, this is the first description of nested multiparametric prediction models for this tumor entity.

Our study sample represents the two patient populations typically triaged to TACE treatment: individuals suffering intermediate stage disease for palliative loco-regional treatment and patients with early stage of disease being poor surgical candidates or undergoing bridging TACE while awaiting liver transplantation. Mean age and gender distribution of our study sample match the typical HCC epidemiology in the Western world [13].

Image-based texture analysis is an emerging methodology to gain additional quantitative information on lesion heterogeneity based on gray-level histograms. It is used to extract and process pixel distribution within a region or interest. Both CT and MRI imaging are generally suitable modalities for analyses, potentially serving as non-invasive imaging biomarkers for prognosis and treatment response [14, 15]. Many studies assessed the feasibility of this method for various tumor entities with promising results, e.g., endometrial cancer [16], pancreatic cancer [17], and non-small cell lung cancer [18].
Several published studies already focused on prediction of therapeutic response of HCCs to transcatheter arterial chemoembolization $[11,12,19,20]$. Strong arterial enhancement, smaller tumor size, and lower homogeneity were found to be significant predictors of a complete response outcome. Our results confirm this observation, since especially the size of a lesion was the parameter with the highest total effect in all prediction models. Tumor heterogeneity, represented by the parameters' uniformity and MPP, also proved to have relevant effects when aiming to predict target response in lesions undergoing primary TACE.

When targeting response prediction to repeated TACE, arterial enhancement seemed to be of less importance though, since it only had an effect in the model predicting progressive disease of RT-lesions, but not in the complete response model. We interpret this finding as resultant change in the underlying lesion vascularization caused by the primary TACE treatment. Due to the desired synergistic effects of vessel blockage and cytotoxic chemotherapeutic agent, the vascular bed of tumors has been damaged sufficiently to impact parenchyma perfusion. Success of repeated TACE hence does not primarily
Table 3 Total effect of lesion size and distinct $\mathrm{CT}$ texture analysis parameters to prediction models

\begin{tabular}{lllll}
\hline Parameter & CR model 1st TACE & PD model 1st TACE & CR model Re-TACE & PD model Re-TACE \\
\hline Area & 0.744 & 0.974 & 0.986 & 0.97 \\
MPP & 0.019 & 0.004 & 0.001 & 0.125 \\
Cirrhosis & 0.145 & 0 & 0.001 & 0 \\
Uniformity & 0.267 & 0.003 & 0.001 & 0 \\
Kurtosis & 0.001 & 0.201 & 0.001 & 0.068 \\
Skewness & 0.003 & 0.078 & 0.001 & 0 \\
Entropy & 0 & 0 & 0.001 & 0 \\
\hline
\end{tabular}

$C R$ complete response, $P D$ progressive disease, $M P P$ mean of positive pixels, $T A C E$ transcatheter arterial chemoembolization 
depend upon good arterial enhancement on pre-therapeutic CT imaging. This change in vascularization after primary TACE treatment is also supported by the fact that we observed significantly lower MPPs in lesions undergoing repeated TACE, compared to lesions scheduled for primary treatment. Therefore, arterial contrast enhancement per se is a less relevant prognostic parameter in HCCs undergoing repeated TACE.

A study that supports our hypothesis was performed by Fujita et al [21] in 2008, who revealed discrepancies between arterial enhancement on pretherapeutic CT scans and uptake of ethiodized oil (Lipiodol). In their study, $14.5 \%$ of tumors with poor to no enhancement on baseline CT images showed, however, moderate to complete accumulation of Lipiodol, emphasizing that success of treatment does not always correlate with lesion enhancement on baseline $\mathrm{CT}$ or hepatic angiography. This likely affects prediction of therapeutic effects of TACE treatment and underlines the benefit of nested multiparametric models which place impacting tissue characteristics in an additive matrix rather than single parameterbased attempts of prediction in patients suffering hepatocellular carcinoma.

Our prediction models and especially decision tree visualization with thresholds for the utilized parameters allowing binary splits are a novel paradigm to transfer CT texture analyses of HCCs into future clinical practice. Decision trees are popular in a wide range of medical and non-medical professions for a variety of reasons, easy interpretability probably being the most important advantage. In contrast to other artificial intelligence models, which also increasingly find their way into clinical practice but are usually based on nontraceable neural networks, decision tree models are comprehensible and reproducible for the user.

Besides clinical relevance, implementability into routine workflows is one of the main challenges when aiming to transfer research innovations into clinical practice. We extracted CT texture parameters from software that radiologists in our department use for longitudinal follow-up imaging in oncologic patients. As the segmentation of target lesions is performed routinely for the radiology report and CT texture parameters are computed automatically, no additional tasks would have to be performed by the interpreting radiologist. This is an advantage over analysis with additional software solutions, resulting in increased workload in daily routine. However, some of these texture analysis tools offer additional parameters, e.g., gray-level co-occurrence matrices, which may further increase prediction model accuracies.

Our study has several limitations. The study sample was rather small; however, by analyzing all treated HCCs of patients, we reached a sufficient number of lesions for modeling. CT scans were performed on two different scanner systems. Resulting possible slight differences in $\mathrm{CT}$ attenuation values may have caused a bias in texture parameters. Since both scanners were however manufactured by the same vendor and we consistently used the same imaging protocol, we rate this possible error of lesser importance. Segmentation of lesions was performed manually which is always prone to errors. Consensus reading of the segmented ROIs by three radiologists, two of them with long-term experience in liver imaging, should have reduced this error to a minimum. Finally, our analysis is based on 2D segmentation on axial image datasets. This is attributed to the analysis according to mRECIST criteria, even though 3D analysis of lesions would possibly have been more accurate in terms of tumor heterogeneity.

In conclusion, our study provides strong evidence that $\mathrm{CT}$ texture analyses of HCC lesions at baseline imaging prior to TACE may be used to accurately predict therapeutic response when using nested multiparametric decision tree models, which are easily understandable for everyone involved in the decision process of triaging patients to TACE.

Supplementary Information The online version contains supplementary material available at https://doi.org/10.1007/s00330-020-07511-3.

Funding Open access funding provided by University of Basel.

\section{Compliance with ethical standards}

Guarantor The scientific guarantor of this publication is Daniel T. Boll.

Conflict of interest The authors of this manuscript declare no relationships with any companies whose products or services may be related to the subject matter of the article.

Statistics and biometry No complex statistical methods were necessary for this paper.

Informed consent Written informed consent was obtained from all subjects (patients) in this study.

Ethical approval Institutional review board approval was obtained.

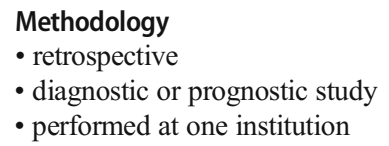

Open Access This article is licensed under a Creative Commons Attribution 4.0 International License, which permits use, sharing, adaptation, distribution and reproduction in any medium or format, as long as you give appropriate credit to the original author(s) and the source, provide a link to the Creative Commons licence, and indicate if changes were made. The images or other third party material in this article are included in the article's Creative Commons licence, unless indicated otherwise in a credit line to the material. If material is not included in the article's Creative Commons licence and your intended use is not permitted by statutory regulation or exceeds the permitted use, you will need to obtain permission directly from the copyright holder. To view a copy of this licence, visit http://creativecommons.org/licenses/by/4.0/. 


\section{References}

1. Tang A, Hallouch O, Chernyak V, Kamaya A, Sirlin CB (2018) Epidemiology of hepatocellular carcinoma: target population for surveillance and diagnosis. Abdom Radiol (NY) 43:13-25. https://doi.org/10.1007/s00261-017-1209-1

2. Villanueva A (2019) Hepatocellular carcinoma. N Engl J Med 380: 1450-1462. https://doi.org/10.1056/NEJMra1713263

3. European Association for the Study of the Liver, European Organisation For Research And Treatment Of Cancer (2012) EASLEORTC clinical practice guidelines: management of hepatocellular carcinoma. J Hepatol 56:908-943. https://doi.org/10.1016/ j.jhep.2011.12.001

4. Hsu CH, Shen YC, Shao YY, Hsu C, Cheng AL (2014) Sorafenib in advanced hepatocellular carcinoma: current status and future perspectives. J Hepatocell Carcinoma 1:85-99. https://doi.org/10. 2147/JHC.S45040

5. Facciorusso A (2018) Drug-eluting beads transarterial chemoembolization for hepatocellular carcinoma: current state of the art. World J Gastroenterol 24:161-169. https://doi.org/10.3748/ wjg.v24.i2.161

6. Vogel A, Cervantes A, Chau I et al (2018) Hepatocellular carcinoma: ESMO clinical practice guidelines for diagnosis, treatment and follow-up. Ann Oncol 29:iv238-iv255. https://doi.org/10.1093/ annonc/mdy308

7. Llovet JM, Real MI, Montaña X et al (2002) Arterial embolisation or chemoembolisation versus symptomatic treatment in patients with unresectable hepatocellular carcinoma: a randomised controlled trial. Lancet 359:1734-1739. https://doi.org/10.1016/ S0140-6736(02)08649-X

8. Lo CM, Ngan H, Tso WK et al (2002) Randomized controlled trial of transarterial Lipiodol chemoembolization for unresectable hepatocellular carcinoma. Hepatology 35:1164-1171. https://doi.org/ 10.1053/jhep.2002.33156

9. Raoul JL, Sangro B, Forner A et al (2011) Evolving strategies for the management of intermediate-stage hepatocellular carcinoma: available evidence and expert opinion on the use of transarterial chemoembolization. Cancer Treat Rev 37:212-220. https://doi. org/10.1016/j.ctrv.2010.07.006

10. Wei J, Wang Z (2019) Establishment of a predictive model for short-term efficacy of transcatheter arterial chemoembolization treatment in hepatocellular carcinoma and its clinical application. J Cancer Res Ther 15:941-946. https://doi.org/10.4103/jcrt.JCRT_ 5219

11. Park HJ, Kim JH, Choi S, Park SJ (2017) Prediction of therapeutic response of hepatocellular carcinoma to transcatheter arterial chemoembolization based on pretherapeutic dynamic CT and textural findings. AJR Am J Roentgenol 209:W211-W221. https:// doi.org/10.2214/AJR.16.17398
12. Yu JY, Zhang HP, Tang ZY et al (2018) Value of texture analysis based on enhanced MRI for predicting an early therapeutic response to transcatheter arterial chemoembolisation combined with highintensity focused ultrasound treatment in hepatocellular carcinoma. Clin Radiol 73:758.e9-758.e18. https://doi.org/10.1016/j.crad. 2018.04.013

13. Yang JD, Hainaut P, Gores GJ, Amadou A, Plymoth A, Roberts LR (2019) A global view of hepatocellular carcinoma: trends, risk, prevention and management. Nat Rev Gastroenterol Hepatol 16: 589-604. https://doi.org/10.1038/s41575-019-0186-y

14. Miles KA, Ganeshan B, Hayball MP (2013) CT texture analysis using the filtration-histogram method: what do the measurements mean? Cancer Imaging 13:400-406. https://doi.org/10.1102/14707330.2013 .9045

15. Lubner MG, Smith AD, Sandrasegaran K, Sahani DV, Pickhardt PJ (2017) CT texture analysis: definitions, applications, biologic correlates, and challenges. Radiographics 37:1483-1503. https://doi. org/10.1148/rg.2017170056

16. Ytre-Hauge S, Dybvik JA, Lundervold A et al (2018) Preoperative tumor texture analysis on MRI predicts high-risk disease and reduced survival in endometrial cancer. J Magn Reson Imaging 48: 1637-1647. https://doi.org/10.1002/jmri.26184

17. Kim HS, Kim YJ, Kim KG, Park JS (2019) Preoperative CT texture features predict prognosis after curative resection in pancreatic cancer. Sci Rep 9:17389. https://doi.org/10.1038/s41598-019-53831-w

18. Ganeshan B, Panayiotou E, Burnand K, Dizdarevic S, Miles K (2012) Tumour heterogeneity in non-small cell lung carcinoma assessed by CT texture analysis: a potential marker of survival. Eur Radiol 22:796-802. https://doi.org/10.1007/s00330-0112319-8

19. Abajian A, Murali N, Savic LJ et al (2018) Predicting treatment response to intra-arterial therapies for hepatocellular carcinoma with the use of supervised machine learning - an artificial intelligence concept. J Vasc Interv Radiol 29:850-857.e1. https://doi.org/ 10.1016/j.jvir.2018.01.769

20. Liu D, Liu F, Xie X et al (2020) Accurate prediction of responses to transarterial chemoembolization for patients with hepatocellular carcinoma by using artificial intelligence in contrast-enhanced ultrasound. Eur Radiol 30:2365-2376. https://doi.org/10.1007/ s00330-019-06553-6

21. Fujita T, Ito K, Tanabe M, Yamatogi S, Sasai H, Matsunaga N (2008) Iodized oil accumulation in hypervascular hepatocellular carcinoma after transcatheter arterial chemoembolization: comparison of imaging findings with CT during hepatic arteriography. J Vasc Interv Radiol 19:333-341. https://doi.org/10.1016/j.jvir.2007. 10.020

Publisher's note Springer Nature remains neutral with regard to jurisdictional claims in published maps and institutional affiliations. 